\title{
A review of the 0.1 reconnection rate problem
}

\author{
P. A. Cassak ${ }^{1, \dagger}$, Y.-H. Liu ${ }^{2}$ and M. A. Shay ${ }^{3}$ \\ ${ }^{1}$ Department of Physics and Astronomy, West Virginia University, Morgantown, WV 26506, USA \\ ${ }^{2}$ Heliophysics Science Division, NASA Goddard Space Flight Center, Greenbelt, MD 20771, USA \\ ${ }^{3}$ Department of Physics and Astronomy, University of Delaware, Newark, DE 19716, USA
}

(Received 1 May 2017; revised 11 August 2017; accepted 11 August 2017)

A long-standing problem in magnetic reconnection is to explain why it tends to proceed at or below a normalized rate of 0.1 . This article gives a review of observational and numerical evidence for this rate and discusses recent theoretical work addressing this problem. Some remaining open questions are summarized.

Key words: astrophysical plasmas, fusion plasma, space plasma physics

\section{Introduction}

Magnetic reconnection is a fundamental plasma process that occurs in many diverse settings including solar physics, magnetospheric physics, astrophysics, heliophysics and fusion energy sciences. It is a key element in the rapid release of magnetic energy in solar flares, geomagnetic substorms, astrophysical transients including anomalous $\mathrm{X}$-ray pulsars and soft gamma repeaters and the sawtooth crash and disruptions in magnetically confined fusion devices (Zweibel \& Yamada 2009). It also plays a crucial role in other plasma processes including the magnetic dynamo and angular momentum transport in accretion disks around compact astrophysical objects (Zweibel \& Yamada 2009) and for dissipation at the end of the turbulent cascade (Servidio et al. 2009, 2011; Cerri \& Califano 2017; Loureiro \& Boldyrev 2017; Mallet, Schekochihin \& Chandran 2017). The key ingredient is that a change in topology of the magnetic field allows for the release of energy stored in the magnetic field into directed kinetic energy and heat.

Observations and numerical simulations in a wide variety of settings suggest that the global rate of magnetic reconnection is approximately 0.1 in normalized units. This means that the electric field pointing out of the reconnection plane that drives the reconnection is near 0.1 when normalized to a properly defined reconnecting magnetic field and Alfvén speed. That this is the case has been known for many years, but developing a theoretical understanding of why has been extremely challenging.

If we already know the typical rate, why is it important to solve this problem? The rate of reconnection is likely related to the efficiency of particle acceleration and heating during the reconnection process, which is a very important aspect of identifying reconnection and studying its effects remotely such as in astrophysical

$†$ Email address for correspondence: Paul.Cassak@mail.wvu.edu 
settings where directly measuring magnetic fields is not possible. At Earth's dayside magnetopause, the reconnection rate is directly related to the efficiency of solar windmagnetospheric coupling and the rate at which the global convection pattern occurs, which is a key aspect of space weather. In magnetically confined fusion devices, the rate of reconnection is related to the rate at which material from the core is ejected during sawteeth. Thus, knowing what controls the reconnection rate in various settings is crucial for applications.

This article presents a review of observational, theoretical and numerical evidence of the normalized reconnection rate being approximately 0.1 . Then, recent work addressing why this is the case is reviewed. Some open questions are then discussed. The reader is also referred to other recent reviews on this topic (Bhattacharjee 2004; Zweibel \& Yamada 2009; Yamada, Kulsrud \& Ji 2010; Cassak \& Shay 2012; Comisso \& Bhattacharjee 2016).

\section{Observational evidence for the 0.1 reconnection rate}

The reconnection rate can be inferred with very basic considerations using plasma parameters observed at macroscopic scales (e.g. Shay et al. 2004). Consider two regions of magnetic field coming together and reconnecting, as shown schematically in figure 1. Let the time over which significant energy release via reconnection occurs be $\Delta t$. Suppose the reconnecting magnetic field has a characteristic strength $B$ threading a region of characteristic radius $R$ and out-of-plane extent $L_{\text {ext }}$, each assumed uniform for simplicity. Then, the magnetic flux processed per unit time by reconnection is $B A / \Delta t$, where $A \sim R L_{\text {ext }}$ as shown by the white surface in the figure. From Faraday's law, this must be associated with an electric field $E$ (the reconnection electric field) extending over a distance $L_{\text {ext }}$ out of the reconnection plane. In SI units, the relation is

$$
E \sim \frac{B A}{L_{\mathrm{ext}} \Delta t} \sim \frac{B R}{\Delta t} .
$$

The reconnection electric field is often presented as a dimensionless quantity that we call $E^{\prime}$, which is normalized by the reconnecting magnetic field $B$ and the Alfvén speed $c_{A}$ based on $B$ and the ambient plasma density $n$, so

$$
E^{\prime}=\frac{E}{B c_{A}} \sim \frac{R}{c_{A} \Delta t} .
$$

There is a geometrical interpretation of this expression. The numerator is the radial distance of magnetic flux reconnected in the time $\Delta t$, and the denominator is the distance that would have been reconnected in the same time if the inflow speed was $c_{A}$. This is consistent with the more commonly quoted form of $E^{\prime}=v_{\text {in }} / c_{A}$, where $v_{\text {in }}$ is the inflow speed.

The reconnected rate computed in this way describes the processing of magnetic flux on a global scale, so we refer to this interchangeably as the global, large-scale or macro-scale reconnection rate. However, we point out that the change of magnetic topology takes place due to dissipation on small scales. Thus, we distinguish the inferred macro-scale reconnection rate from the reconnection rate measured locally near the reconnection site. This is computed by normalizing to the magnetic field and plasma parameters immediately upstream of the dissipation region, so it need not be the same as the global reconnection rate. We interchangeably refer to the local rate as the small-scale or micro-scale reconnection rate. We are now prepared to estimate the global reconnection rate from (2.2) for various settings where reconnection commonly occurs. 


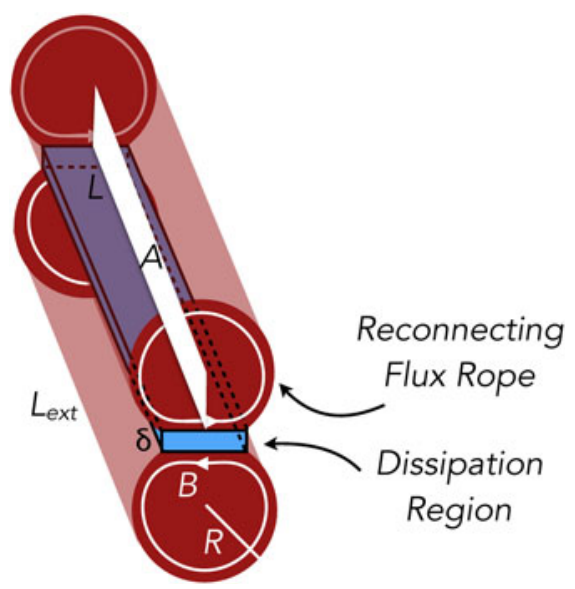

FIGURE 1. Sketch of two reconnecting flux ropes in red with reconnecting magnetic field lines in white. The flux rope radius $R$ and out-of-plane extent $L_{\text {ext }}$ are shown. The dissipation region of thickness $\delta$ and length $L$ is in blue. The white surface of area $A$ denotes the location of the magnetic field that reconnects in a time $\Delta t$.

\subsection{Solar flares}

For solar flares, we take plasma parameters from Priest \& Forbes (2002). In large solar flares releasing over $10^{24} \mathrm{~J}$, energy is released beginning with an impulsive phase that lasts approximately $\Delta t \simeq 100 \mathrm{~s}$, although energy release continues to a lesser extent over much longer times. A magnetic field of strength $B \simeq 100 \mathrm{G}=0.01 \mathrm{~T}$ threads flux tubes of radius $R \sim 3 \times 10^{7} \mathrm{~m}$ and extent $L_{\text {ext }} \sim 10^{8} \mathrm{~m}$; this provides more than enough magnetic energy $\left(B^{2} / 2 \mu_{0}\right) \pi R^{2} L_{\text {ext }}$ to power the flare. Using these parameters, the inferred absolute reconnection rate from $(2.1)$ is $E \simeq 3000 \mathrm{~V} \mathrm{~m}^{-1}$, and the normalized global reconnection rate from (2.2) using $c_{A} \simeq 4 \times 10^{6} \mathrm{~m} \mathrm{~s}^{-1}$ (based on a density of $3 \times 10^{15} \mathrm{~m}^{-3}$ ) is

$$
E_{\text {flare }}^{\prime}=0.075 \text {. }
$$

\subsection{Geomagnetic substorms}

In Earth's magnetotail during geomagnetic substorms, the expansion phase takes place over a time scale of approximately $30 \mathrm{~min}$ (McPherron 1970). The relevant magnetotail parameters are a lobe magnetic field $B \simeq 20 \mathrm{nT}$, the distance from the plasma sheet to the magnetopause is $R \simeq 15 R_{E}$ and the cross-tail extent is $L_{\text {ext }} \simeq 30 R_{E}$ (Axford, Petschek \& Siscoe 1965), where $R_{E}$ is the Earth's radius. The absolute and normalized global reconnection rate necessary to reconnect the magnetotail in the observed time, using $c_{A} \simeq 10^{6} \mathrm{~m} \mathrm{~s}^{-1}$ based on a density of $0.1 \mathrm{~cm}^{-3}=10^{5} \mathrm{~m}^{-3}$ in (2.1) and (2.2), is $E \simeq 1.1 \mathrm{mV} \mathrm{m}^{-1}$ (corresponding to a cross-tail potential of $200 \mathrm{kV}$ ) and

$$
E_{\text {substorm }}^{\prime} \simeq 0.053 \text {. }
$$

The similarity of the inferred flare and substorm normalized reconnection rates prompted Parker (1973) to say 'altogether, the observations of both solar and magnetospheric activity suggest that reconnection rates are "universally" of the general order of magnitude of $0.1 V_{A}$ '. 


\subsection{The sawtooth crash}

In the sawtooth crash in magnetically confined fusion devices (von Goeler, Stodiek \& Sautoff 1974), the absolute global reconnection rate $E$ should depend on the size, shape and plasma parameters in the device in question. We are unaware of any comparative studies of normalized reconnection rates in sawteeth. However, to get a feel for a reasonable rate in a modern device, we consider a shot in the Mega Ampere Spherical Tokamak (MAST) described by Chapman et al. (2010), where the sawtooth crash time is $\Delta t \simeq 120 \mu \mathrm{s}$. The relevant reconnection parameters were extracted by Beidler \& Cassak (2011): the major radius is $R_{0}=0.85 \mathrm{~m}$, so $L_{\mathrm{ext}} \simeq 2 \pi R_{0}$; the $q=1$ rational surface where reconnection occurs is at $R=0.32 \mathrm{~m}$, where $q$ is the safety factor; the reconnecting (auxiliary) magnetic field at the upstream edge of the ion diffusion region (at a distance of an ion Larmor radius upstream of the auxiliary field reversal) is $B \simeq 6.7 \mathrm{mT}$, the corresponding Alfvén speed is $c_{A} \simeq 13 \mathrm{~km} \mathrm{~s}^{-1}$. The associated global reconnection rate for processing the whole core, from (2.2), is

$$
E_{\text {sawtooth }}^{\prime} \simeq 0.21 \text {. }
$$

This value is close to the flare and substorm results. While a single shot in a single device does not imply that all devices have the same rate for all shots, it supports the notion that the normalized reconnection rate is similar across many settings.

\subsection{More direct observations}

Each of these examples provide an indirect measurement of the global reconnection rate. With modern observations, other approaches to infer the global reconnection rate have become available. Magnetic fields at the reconnection site in solar flares cannot be directly measured, but the speed with which ribbons at the footpoints of two ribbon flares separate is related to the rate at which magnetic flux is reconnected higher up in the corona. Observations give normalized rates of 0.001-0.2 (Ohyama \& Shibata 1998; Yokoyama et al. 2001; Isobe et al. 2002; Qiu et al. 2002; Fletcher, Pollack \& Potts 2004; Isobe, Takasaki \& Shibata 2005; Lin et al. 2005), although it should be cautioned that uncertainties in the magnetic fields to normalize to are quite large. For the magnetosphere, reconnection rates can be inferred from the convective electric field; they are not too different than 0.1 at the dayside (Mozer \& Pritchett 2010; Wang et al. 2015) and magnetotail (Blanchard et al. 1996), although again uncertainties are large because there is uncertainty associated with defining the reconnection plane using techniques such as minimum variance analysis and its variants.

A direct measurement of the reconnection electric field locally has been challenging because of its small size and the same uncertainties related to finding the appropriate reference frame. It has been inferred in laboratory experiments; a value of 0.35 was reported in the Versatile Toroidal Facility (Egedal et al. 2007). However, using the Magnetospheric Multiscale (MMS) satellites (Burch et al. 2016a), the high frequency electric field for an event was filtered out revealing a DC out-of-plane field in reasonable agreement with 0.1 (Chen et al. 2017). Thus, there appears to be indirect and direct observational support that the rate typically is approximately equal to, and potentially less than, 0.1 .

\section{Early numerical and theoretical efforts}

Before reconnection was discovered (Dungey 1953), there was no explanation of how magnetic energy could be converted so rapidly. Magnetic diffusion was orders 


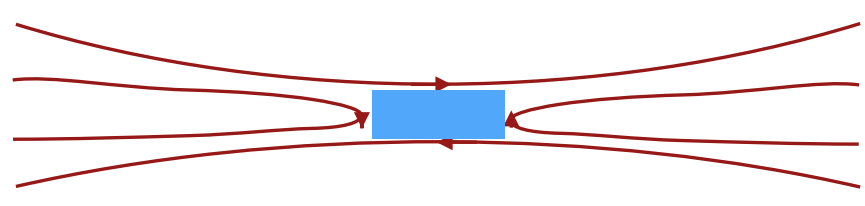

FIGURE 2. Sketch of reconnecting and reconnected magnetic field lines in red, with the dissipation region denoted by the blue rectangle.

of magnitude too slow. Before treating how reconnection can be fast enough to explain the observed rates, we point out that theoretical and numerical studies on this problem have typically been performed using simplified systems. Unless otherwise noted, the treatment here is for two-dimensional reconnection with anti-parallel reconnecting magnetic fields (i.e. no 'guide' magnetic field), as sketched in figure 2. (The presence of a uniform guide field is not expected to qualitatively or quantitatively alter the considerations that follow, except as discussed in $\S 5$.) We assume the two sides of the reconnection region are symmetric and isotropic, and the upstream plasma has no upstream bulk flow and is laminar as opposed to turbulent. We also only treat spontaneous reconnection in the steady state, meaning the resultant rate from perturbing a current sheet and waiting until the reconnection reaches a quasi-steady state, as opposed to continually forcing the reconnection with given upstream conditions. The process is studied locally near the dissipation region, and most studies have not attempted to relate the local physics to global considerations from far upstream of the reconnection site. We make these assumptions not with the idea that all reconnection satisfies these restrictions, but that the process is easier to study in this limit and simulations reveal that it contains sufficient physics to reproduce the 0.1 reconnection rate.

Parker (1957) performed a scaling analysis of the reconnection process providing what we now call Sweet-Parker theory; based on conservation of mass, energy and magnetic flux, he showed that the reconnection rate scales as

$$
E \sim \frac{\delta}{L} v_{\text {out }} B
$$

where $\delta$ is the dissipation region thickness in the inflow direction and $L$ is its length in the outflow direction, $v_{\text {out }}$ is the outflow speed (which scales as the Alfvén speed $c_{A}$ ), $B$ is the reconnecting magnetic field strength, and he showed that $\delta / L \sim 1 / S^{1 / 2}$ when the mechanism allowing the magnetic topology to change is a uniform resistivity, where $S$ is the Lundquist number. The local reconnection rate, normalized to $B$ and $c_{A}=v_{\text {out }}$, is $E^{\prime} \sim \delta / L$. He originally suggested reconnection could be fast enough to explain flares, but he soon realized that the prediction was too slow (Parker 1963). Physically, the failure of the Sweet-Parker model is that the exhaust region closes down into an elongated region. Since the outflow is constrained to leave at the Alfvén speed, conservation of mass requires the inflow speed to be small. Despite the rate being too small to explain observations, it is still a physically correct model that accurately describes resistive reconnection with sufficiently high resistivity. It has been confirmed in simulations (Uzdensky \& Kulsrud 2000; Cassak, Shay \& Drake 2005) and experiments (Ji et al. 1998; Trintchouk et al. 2003; Furno et al. 2005). The disagreement with observed rates spurred research to figure out why reconnection is faster than what is predicted by the Sweet-Parker model. We point out in passing that it was later determined that Sweet-Parker reconnection is untenable for large 
enough $S$; instead the Sweet-Parker layer breaks up producing secondary islands, which fundamentally changes the rate. Since our treatment here is only for a single $\mathrm{X}$-line, we postpone a discussion of this important effect to $\S 5$.

The Petschek (1964) model predicts local reconnection rates closer to the observationally inferred global rate. In the Petschek model, the curved magnetic fields in the exhaust straighten out, which produces slow shocks propagating out along the magnetic field that accelerate the plasma into the outflow jet and provide a more open exhaust region. The dissipation region in the Petschek model is more localized than in the Sweet-Parker model. The model caused excitement that the problem was solved, but work much later benefiting from numerical simulations revealed that the Petschek model is not self-consistent in the magnetohydrodynamic (MHD) model with a uniform resistivity (Biskamp 1986; Uzdensky \& Kulsrud 2000). However, other models (as described below) do produce shocks bounding open exhausts (Liu, Drake \& Swisdak 2012; Innocenti et al. 2015), so many aspects of the Petschek model are believed to be essentially correct.

An advance occurred when it was realized that a fluid model with a localized (also called 'anomalous') resistivity could produce Petschek-like reconnection with the associated high rates (Ugai \& Tsuda 1977; Sato \& Hayashi 1979). Physically, a region of stronger diffusion near the reconnection site causes the magnetic field line to bend in, which gives an open outflow exhaust (Kulsrud 2001). There have been efforts to identify physical causes of anomalous resistivity (e.g. Huba, Gladd \& Papadopoulos 1977; Ugai 1984; Strauss 1987), but it is not well established that the reconnection rate can be explained through this mechanism.

An alternate approach considers reconnection in essentially collisionless systems, for which the scale of collisional diffusion is much smaller than electron and ion gyroradius scales. Calculations (Drake \& Lee 1977; Terasawa 1983; Hassam 1984) and simulations (Aydemir 1991) suggested that the linear phase of collisionless reconnection (the tearing mode) was much faster than its collisional counterpart. Numerical studies of steady-state collisionless reconnection in which the electronto-ion mass ratio (Shay \& Drake 1998; Hesse et al. 1999; Shay, Drake \& Swisdak 2007) and ratio of system size to ion inertial length (Shay et al. 1999) were varied found that the local reconnection rate was approximately 0.1 independent of those two quantities. This prompted the idea that collisionless reconnection had a 'universal' rate (Shay et al. 1999). The Geospace Environment Modeling (GEM) Challenge study compared simulations with different models, and found that all models with the Hall term (two fluid, hybrid and full particle in cell) had rates comparable to the 0.1 value (Birn et al. 2001). Many concluded from this that the Hall term was the cause of reconnection having a rate of 0.1 . While no there is no first-principles theory showing the reconnection rate is 0.1 , it was suggested that the dispersive behaviour of the Hall term gives rise to faster flows at smaller scales, which causes the outflow exhausts to open up as is necessary for reconnection rates faster than Sweet-Parker (Mandt, Denton \& Drake 1994; Rogers et al. 2001; Drake, Shay \& Swisdak 2008; Cassak, Shay \& Drake 2010). It should be pointed out that the Hall term is sufficient to give rates near 0.1 , although it is of course not necessary (since it was already known that the same rates arose in MHD with a localized resistivity).

The situation became more complicated when other systems were also found to have a similar local reconnection rate. An electron-positron plasma is potentially important for some astrophysical applications including pulsar winds and extragalactic jets, but is also important for fundamental physics because the Hall term in such a plasma is not 
present when the electron and ion temperatures are equal (Bessho \& Bhattacharjee 2005). Simulations have revealed that the rate in such a system is also of order 0.1 (Bessho \& Bhattacharjee 2005, 2007; Daughton \& Karimabadi 2007; Hesse \& Zenitani 2007; Swisdak, Liu \& Drake 2008; Zenitani \& Hesse 2008). The mechanism causing the reconnection rate to be close to 0.1 remains controversial; three different possible mechanisms include secondary islands (Daughton \& Karimabadi 2007), off-diagonal elements of the pressure tensor (Bessho \& Bhattacharjee 2007) and the Weibel instability (Swisdak et al. 2008). When there is a large out-of-plane (guide) magnetic field, the Hall term can become inactive; reconnection in such a regime also has a similar rate (Liu et al. 2014; Cassak et al. 2015; Stanier et al. 2015b). These results confirm that the Hall term and their associated dispersive waves are not necessary to get reconnection rates near 0.1 .

The reconnection rate has also been studied in relativistic reconnection, often with astrophysical applications in mind. Most numerical studies have been for electron-positron plasmas because of the reduced numerical expense. The global reconnection rate continues to be close to 0.1 (Bessho \& Bhattacharjee 2012; Liu et al. 2015; Sironi, Giannios \& Petropoulou 2016), although interestingly the local rate can approach 1 (Liu et al. 2015). Relativistic electron-proton reconnection has a similar global rate as well (Melzani et al. 2014). A similar rate was found in particle-in-cell (PIC) simulations of reconnection in high energy density laser plasmas (Fox, Bhattacharjee \& Germaschewski 2011). In summary, the global reconnection rate in a wide variety of systems described by different physical models and simulated with different simulation tools has a normalized reconnection rate of approximately 0.1 , but it is not clear why this is the case. We must conclude that either multiple disparate mechanisms mysteriously give rise to the same rate, or there is something more fundamental causing the similar rates.

\section{Recent insights}

The fact that the (local) reconnection rate is completely altered by going from a uniform to a localized resistivity within the MHD model and the similar stark differences between reconnection rates for systems with and without the Hall term undoubtedly led researchers to focus on the physics of the dissipation allowing magnetic topology to change (i.e. local physics), to try to solve the 0.1 reconnection rate problem. However, the evidence discussed in the previous section suggests this was a misleading approach. With the exception of reconnection in MHD with a uniform resistivity (with or without secondary islands), the global reconnection rate in all models tends to be the same. This suggests that the reconnection rate is set not by the local (micro-scale) physics allowing the dissipation, but is due to constraints at the MHD (macro-) scale since the only aspect all different forms of reconnection have is that they match up with MHD at the large scales.

Very simple considerations are sufficient to see how large-scale physics could limit the global reconnection rate. In the Sweet-Parker model, the local reconnection rate scales like the aspect ratio of the diffusion region, as shown in (3.1). This suggests that elongated layers are associated with slower reconnection, and opening up the exhaust angle increases the reconnection rate. This is certainly true of Sweet-Parker reconnection, but there is a limitation. If one continues to open up the exhaust, one eventually reaches a system where the exhaust opening angle is $90^{\circ}$. Rather than having a normalized reconnection rate of 1 (with the inflow speed equal to the Alfvén speed) as would be predicted by $E^{\prime}=\delta / L$, one actually gets $E^{\prime}=0$ ! This is the case 
(a)

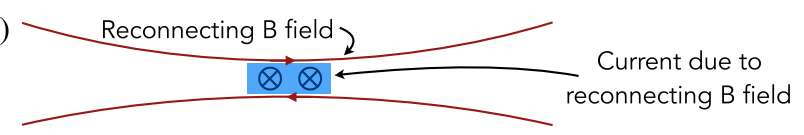

(b)

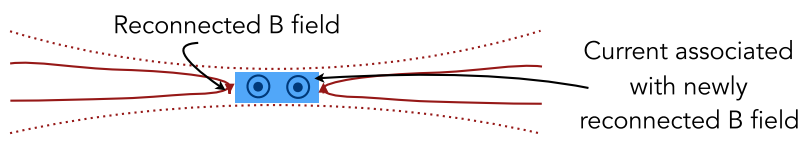

FIgURE 3. (a) Magnetic field lines in red before they reconnect, with their associated current in the dissipation region (in blue) into the page. (b) Magnetic field lines after they reconnect; the current due to the newly reconnected field lines opposes the current in panel $(a)$. This motivates why reconnection cannot be made arbitrarily fast.

because the free energy available for release decreases with increasing opening angle (Jemella et al. 2003), and it vanishes in the limit of a $90^{\circ}$ opening angle (Cassak and Shay, unpublished, 2008). Equivalently, the symmetry between the inflow and outflow regions for a $90^{\circ}$ opening angle prevents spontaneous reconnection (Comisso \& Bhattacharjee 2016). This implies there is a limitation due to the large-scale physics that prevents global reconnection rates of reaching 1. There must be a maximum global reconnection rate that is faster than Sweet-Parker reconnection but slower than 1.

What is the physical reason that the global reconnection rate decreases when the exhaust opening angle is increased past some threshold value towards $90^{\circ}$ ? A qualitative way to understand this is to consider oppositely directed magnetic fields that are about to reconnect, as in figure $3(a)$. The spatial variation in the upstream (horizontal) field requires an out-of-plane current, in this case into the page. Once those magnetic field lines reconnect, as in figure 3(b), reconnected (vertical) fields appear. From Ampére's law, the current density associated with the newly reconnected components of the magnetic field is out of the plane, but it opposes the direction of the background current. If one were to make reconnection faster, the reconnected magnetic fields would be stronger, so the current associated with the reconnected magnetic fields would become larger. This weakens the overall current, which can be seen directly from Ampére's law evaluated at the blue box in figure 3 (modified from Hesse et al. 2009); evaluating the integral form $\oint \boldsymbol{B} \cdot \mathrm{d} \boldsymbol{l}=\mu_{0} \int \boldsymbol{J} \cdot \mathrm{d} \boldsymbol{A}$, where $\boldsymbol{B}$ is the magnetic field, $\boldsymbol{J}$ is the current density, $\mathrm{d} \boldsymbol{A}$ is the area element directed out of the page, and $\mathrm{d} \boldsymbol{l}$ is an infinitesimal path length around the exterior in a clockwise direction gives

$$
J_{z}=\frac{1}{\mu_{0}}\left(\frac{B_{x}}{\delta}-\frac{B_{y}}{L}\right),
$$

where $B_{x}$ is the magnitude of the horizontal field at the top and bottom borders of the box, $B_{y}$ is the magnitude of the vertical field at the left and right edges, $J_{z}$ is the out-of-plane current density and $\delta$ and $L$ are the half-thickness and length of the blue box. In the incompressible limit, which is sufficient for our purposes, $B_{y} / B_{x} \sim \delta / L$, so (4.1) becomes

$$
J_{z}=\frac{B_{x}}{\mu_{0} \delta}\left(1-\frac{\delta^{2}}{L^{2}}\right) .
$$

As $B_{y} \rightarrow B_{x}$ (i.e. $\delta \rightarrow L$ ), then $J_{z}$ decreases, approaching zero as $B_{y}$ equals $B_{x}$. As the current decreases, the $\boldsymbol{J} \cdot \boldsymbol{E}$ work done by the electric field associated with 


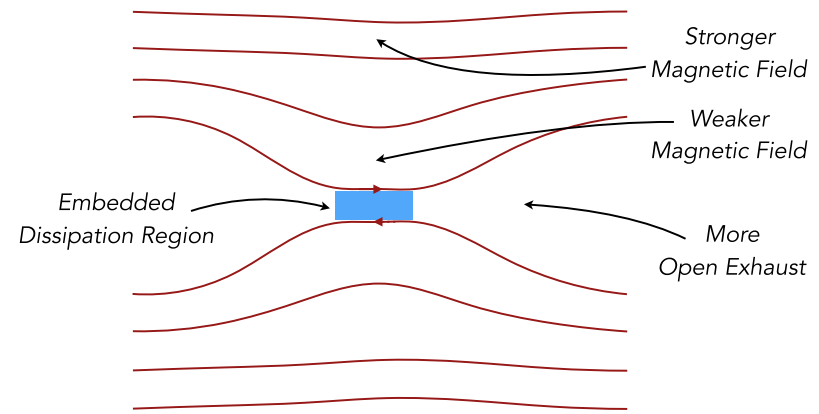

FIGURE 4. Sketch of the reconnection region if the opening angle of the exhaust were to be made more open. This sketch motivates that the magnetic field at the dissipation region becomes weaker because the dissipation region remains at micro-scales, after Liu et al. (2017).

the dissipation required to change magnetic topology decreases, and the reconnection slows. The limit where the current due to the reconnected field equals the current due to the reconnecting field would have a reconnection rate of zero, which is the case of a $90^{\circ}$ exhaust opening angle.

As the opening angle increases, the outflow speed decreases (Hesse et al. 2009). A scaling analysis of the $x$ component of the momentum equation that balances the convection term $\rho(\boldsymbol{v} \cdot \nabla) v_{x}$ with the $(\boldsymbol{J} \times \boldsymbol{B})_{x}=J_{z} B_{y}$ (Lorentz) force reveals that the outflow speed $v_{x}=v_{\text {out }}$ scales like (Hesse et al. 2009)

$$
v_{\mathrm{out}} \sim c_{A} \sqrt{1-\frac{\delta^{2}}{L^{2}}} .
$$

Physically, the reason the outflow speed decreases with increasing opening angle is because the magnetic pressure term (related to the second term in (4.1)) increases with increasing $\delta$ and cancels out the magnetic tension force (related to the first term). Since the reconnection rate is proportional to $v_{\text {out }}$, as shown in (3.1), this suggests the reconnection rate vanishes for an opening angle of $90^{\circ}$. However, using this expression in (3.1) predicts a maximum rate near 0.4 , which is noticeably larger than the reconnection rate obtained in simulations or observations.

A potentially important development came recently, when it was argued that there is a second reason that the reconnection rate decreases when increasing the opening angle (Liu et al. 2017). When the opening angle is increased, the thickness of the current sheet can exceed the length scales associated with the micro-scale processes that allow magnetic topology to change. This happens because the micro-scale physics typically have a fixed scale which defines the region over which they are important. For example, collisionless anti-parallel reconnection in an electron-proton plasma has micro-scales set by the ion and electron inertial scales $d_{i}=c / \omega_{p i}$ and $d_{e}=c / \omega_{p e}$ (Sonnerup 1979). The dissipation region, therefore, cannot respond by getting arbitrarily thick. Instead, the system responds by developing a three-scale structure with the micro-scale set by dissipation physics, the macro-scale set externally, and an intermediate meso-scale that joins the two. The micro-scale dissipation region becomes embedded within the meso-scale structure.

This has important consequences. For systems with low plasma $\beta$, the reconnecting magnetic field immediately upstream of the dissipation region gets weaker, as is sketched in figure 4. Physically, this occurs because the curvature force away from 
the current sheet in the upstream region must be nearly balanced by the magnetic pressure gradient force towards the current sheet. This implies the reconnecting magnetic field at the reconnection site is weaker than the large-scale magnetic field, so the local magnetic field $B$ driving the outflow is weaker. This decreases the reconnection rate, as seen in (3.1), both because the reconnection rate is directly proportional to $B$ and because the Alfvén speed is directly proportional to $B$. A short calculation on very general grounds showed that the local magnetic field $B$ is

$$
B \sim B_{0}\left(\frac{1-\delta^{2} / L^{2}}{1+\delta^{2} / L^{2}}\right),
$$

where $B_{0}$ is the asymptotic macro-scale magnetic field. When convolved with the outflow speed in (4.3) to find the reconnection rate in (3.1), it is found that the maximum in the normalized global reconnection rate is $E^{\prime} \simeq 0.2$ (Liu et al. 2017), much closer to the observed maximum rate. It was further shown that the weakening of the upstream reconnecting field is more important to the scaling than the weakening of the outflow speed. The results compared favourably with PIC simulations of both non-relativistic electron-proton and relativistic electron-positron plasmas (Liu et al. 2017). This result presented a new motivation for why the reconnection rate is close to, and bounded above by, 0.1 .

In summary, these new insights suggest that the constraints of energy release at the meso- and macro-scale play a crucial role in setting the maximum global reconnection rate. An appealing aspect of this approach is that it provides a natural explanation for why the global reconnection rate is the same in the varied numerical systems where a rate of 0.1 has been obtained with vastly different micro-scale physics. It also properly implies that the reconnection rate can only be between 0 and 1 , which is not the case in the Petschek (1964) model.

\section{Some open questions}

The previous section does not imply the 0.1 problem is solved. We outline a number of open questions:

(i) Perhaps the most important unsolved problem is, even continuing to make the simplifying assumptions made here, why is the global reconnection rate not always the maximum rate near 0.1 ? There is a distinct difference between collisionless reconnection and reconnection in MHD with anomalous resistivity on the one hand, and reconnection in MHD with a uniform resistivity on the other. For the latter, if the resistivity is above a threshold (where the Lundquist number $S$ based on the length of the current sheet is below $10^{4}$ (Biskamp 1986; Loureiro, Schekochihin \& Cowley 2007)), collisional reconnection proceeds as outlined by the Sweet-Parker model rather than with a reconnection rate of 0.1 . If the resistivity is uniform but below the same threshold, secondary islands spontaneously arise and the normalized reconnection rate is (Bhattacharjee et al. 2009; Cassak, Shay \& Drake 2009; Huang \& Bhattacharjee 2010)

$$
E^{\prime} \simeq 0.01 \text {. }
$$

It is important to point out that there is a distinct difference between the 0.01 rate when secondary islands are present in resistive reconnection and the 0.1 rate of collisionless reconnection (Daughton et al. 2009; Shepherd \& Cassak 2010). 
Why does Sweet-Parker reconnection not proceed at the maximum rate? When secondary islands arise and make reconnection faster, why is the reconnection rate limited by 0.01 instead of 0.1 ? Answering both of these questions is crucial to a full understanding of the 0.1 problem.

(ii) A related question is the following: is the resistive-MHD description of magnetic reconnection ever valid other than potentially transiently? Since the observed reconnection rates are close to 0.1 , does that imply that the slower modes of Sweet-Parker reconnection and resistive reconnection with secondary islands are not the dominant mode in the release of magnetic energy by reconnection? It has been suggested that resistive reconnection could potentially occur at the early phase of the energy release, but that a transition to faster reconnection is necessary for the rapid energy release (Shibata \& Tanuma 2001; Cassak et al. 2005; Uzdensky 2007) and it has been pointed out that reconnection electric fields are significantly stronger than the Dreicer electric field so that classical resistivity is not possible (e.g. Cassak \& Drake 2013), while others argue that resistive effects are sufficient and can explain the energy release.

(iii) How does the reconnection rate change when we start relaxing our assumptions? When the upstream plasmas are asymmetric, the reconnection rate remains 0.1 (Cassak \& Shay 2008; Malakit et al. 2010) when properly normalized (Cassak $\&$ Shay 2007). However, diamagnetic effects in the presence of a guide field (Swisdak et al. 2003; Liu \& Hesse 2016) or the presence of an upstream flow (Cassak \& Otto 2011; Doss et al. 2015) can reduce the reconnection rate and even prevent reconnection all together. The effect of the diamagnetic drift is interesting because it is a finite Larmor radius effect that is not present in the MHD model. Thus, local finite Larmor radius effects can also impact the global reconnection rate, which along with the first bullet point is another example that the global reconnection rate is not purely set by macro-scale physics like the model in the previous section might suggest. How does the slowing of reconnection due to diamagnetic effects fit in to the picture of maximizing the global rate of reconnection?

(iv) How does the local reconnection picture described in the simplified models fit into global large-scale physics in the context of applications? This is an important problem even in more complicated idealized reconnection studies than the ones discussed here. In the island coalescence problem, two like current channels attract each other, causing reconnection in the magnetic fields between them. If the current channels are much bigger than kinetic scales, the flux ropes can bounce off each other (Karimabadi et al. 2011; Ng et al. 2015; Stanier et al. $2015 a$ ) because the time scale for reconnection can become longer than the time scale of the interaction. The global reconnection rate in that case is quite small, i.e. reconnection does not always proceed at the 0.1 rate discussed here. However, if the local reconnection rate in the embedded current sheet (Shay et al. 2004) is measured, the local reconnection rate is 0.1 (Karimabadi et al. 2011). Such questions are crucial for applications of reconnection to all settings, including solar, magnetospheric, astrophysics and fusion. For example, it is particularly important for flux ropes in the corona which are far larger than kinetic scales. How can a theory be developed to bridge the macro- and micro-scales? The approaches by Simakov, Chacón \& Knoll (2006) and Liu et al. (2017) might be useful.

(v) Why does reconnection not always proceed, i.e. what causes reconnection onset? For example, in the corona before a flare and the magnetotail before a substorm, 
current sheets must exist because they are associated with free energy, but large-scale reconnection does not occur. Large amounts of energy could not be stored if the current sheets were to always reconnect with a rate of 0.1 (Dahlburg, Klimchuk \& Antiochos 2005) or even 0.01 (Cassak \& Drake 2009). What prevents the release of energy at the maximum allowable rate during times when energy is being stored?

(vi) Why do results based on idealized two-dimensional (2-D) systems seem to work so well in producing a rate that is consistent with the rates inferred from global observations which are three-dimensional? Is reconnection really typically quasi-two-dimensional? There are many examples of successful direct comparisons between magnetospheric satellite data and 2-D numerical simulations, which suggests reconnection may be a quasi-2-D process. More recently, MMS satellites have observed strong electric fields that arise in 3-D simulations but not in two dimensions (e.g. Ergun et al. 2016; Burch et al. $2016 b$ ), so clearly 3-D effects can be important. However, when quasi-2-D reconnection is averaged over the out-of-plane direction even in a system with complicated reconnection patterns, the same reconnection rate near 0.1 appears (Daughton, plenary talk at 2016 US-Japan Meeting on Magnetic Reconnection), so it is not clear how the observed 3-D structure impacts the global reconnection rate. Can the global reconnection rate in 3-D systems also be explained as a result of constraints on the global energy release or does it require more general approaches (Pontin 2011; Boozer 2013)? More fundamentally, what conditions must be met for the 2-D model to be accurate? In one study, reconnection with current sheets of small extent in the out-of-plane direction had lower reconnection rates than 2-D reconnection, suggesting that reconnection is more energetically favourable when it is quasi-two-dimensional than fully three-dimensional (Meyer 2013). This was attributed to the ends of the X-line being energy sinks. Significantly more work is needed on this problem. Another interesting open question is: how does upstream turbulence (Matthaeus \& Lamkin 1985; Smith et al. 2004) impact the global reconnection rate?

\section{Acknowledgements}

The authors acknowledge wonderful conversations on this problem with many community members over the years. We acknowledge support from NSF grant AGS-1460037 (P.A.C.) and NASA grants NNX16AG76G (P.A.C.), NNX16AG75G (Y.-H.L.) and NNX15AW58G (M.A.S.). This manuscript was prepared in advance of the first Journal of Plasma Physics Frontiers in Plasma Physics Conference in anticipation of lively conversations.

\section{REFERENCES}

Axford, W. I., Petschek, H. E. \& Siscoe, G. L. 1965 Tail of the magnetosphere. J. Geophys. Res. 70, 1231.

AYdEMIR, A. Y. 1991 Linear studies of $m=1$ modes in high-temperature plasmas with a four-field model. Phys. Fluids B 3 (11), 3025.

BEIDLER, M. T. \& CASSAK, P. A. 2011 Model for incomplete reconnection in sawtooth crashes. Phys. Rev. Lett. 107, 255002.

Bessho, N. \& BhattacharJee, A. 2005 Collisionless reconnection in an electron-positron plasma. Phys. Rev. Lett. 95, 245001. 
Bessho, N. \& BhattacharJee, A. 2007 Fast collisionless reconnection in electron-positron plasmas. Phys. Plasmas 14, 056503.

Bessho, N. \& Bhattacharjee, A. 2012 Fast magnetic reconnection and particle acceleration in relativistic low-density electron-positron plasmas without guide field. Astrophys. J. 750, 129.

BhattacharJeE, A. 2004 Impulsive magnetic reconnection in the earth's magnetotail and the solar corona. Annu. Rev. Astron. Astrophys. 42, 365-384.

Bhattacharjee, A., Huang, Y.-M., Yang, H. \& Rogers, B. 2009 Fast reconnection in highLundquist-number plasmas due to secondary tearing instabilities. Phys. Plasmas 16, 112102.

Birn, J., Drake, J. F., Shay, M. A., Rogers, B. N., Denton, R. E., Hesse, M., Kuznetsova, M., Ma, Z. W., Bhattacharjee, A., Отto, A. et al. 2001 GEM magnetic reconnection challenge. J. Geophys. Res. 106, 3715.

Biskamp, D. 1986 Magnetic reconnection via current sheets. Phys. Fluids 29, 1520.

Blanchard, G. T., Lyons, L. R., de la Beaujardiére, O., Doe, R. A. \& Mendillo, M. 1996 Measurement of the magnetotail reconnection rate. J. Geophys. Res. 101, 15265.

Boozer, A. H. 2013 Model of magnetic reconnection in space and astrophysical plasmas. Phys. Plasmas 20, 032903.

Burch, J. L., Moore, T. E., Torbert, R. B. \& Giles, B. L. $2016 a$ Magnetospheric multiscale overview and science objectives. Space Sci. Rev. 199, 5.

Burch, J. L., Torbert, R. B., Phan, T. D., Chen, L.-J., Moore, T. E., Ergun, R. E., Eastwood, J. P., Gershman, D. J., Cassak, P. A., Argall, M. R. et al. 2016b Electronscale measurements of magnetic reconnection in space. Science 352, 6290.

Cassak, P. A., Baylor, R. N., Fermo, R. L., Beidler, M. T., Shay, M. A., Swisdak, M., DRAKe, J. F. \& KARIMABADI, H. 2015 Fast magnetic reconnection due to anisotropic electron pressure. Phys. Plasmas 22, 020705.

CAssak, P. A. \& Drake, J. F. 2009 The impact of microscopic magnetic reconnection on pre-flare energy storage. Astrophys. J. Lett. 707, L158.

CAssak, P. A. \& Drake, J. F. 2013 On phase diagrams of magnetic reconnection. Phys. Plasmas 20, 061207.

CAssak, P. A. \& ОTto, A. 2011 Scaling of the magnetic reconnection rate with symmetric shear flow. Phys. Plasmas 18, 074501.

CASSAK, P. A. \& Shay, M. A. 2007 Scaling of asymmetric magnetic reconnection: General theory and collisional simulations. Phys. Plasmas 14, 102114.

CAssak, P. A. \& ShaY, M. A. 2008 Scaling of asymmetric Hall reconnection. Geophys. Res. Lett. 35, L19102.

CASSAK, P. A. \& ShaY, M. A. 2012 Magnetic reconnection for coronal conditions: reconnection rates, secondary islands and onset. Space Sci. Rev. 172, 283.

Cassak, P. A., Shay, M. A. \& Drake, J. F. 2005 Catastrophe model for fast magnetic reconnection onset. Phys. Rev. Lett. 95, 235002.

CAssak, P. A., Shay, M. A. \& DraKe, J. F. 2009 Scaling of Sweet-Parker reconnection with secondary islands. Phys. Plasmas 16, 102702.

Cassak, P. A., Shay, M. A. \& Drake, J. F. 2010 A saddle-node bifurcation model of magnetic reconnection onset. Phys. Plasmas 17, 062105.

Cerri, S. S. \& CAlifano, F. 2017 Reconnection and small-scale fields in 2d-3v hybrid-kinetic driven turbulence simulations. New J. Phys. 19, 025007.

Chapman, I. T., Scannell, R., Cooper, W. A., Graves, J. P., Hastie, R. J., Naylor, G. \& ZocCO, A. 2010 Magnetic reconnection triggering magnetohydrodynamic instabilities during a sawtooth crash in a tokamak plasma. Phys. Rev. Lett. 105, 255002.

Chen, L.-J., Hesse, M., Wang, S., Gershman, D., Ergun, R. E., Burch, J., Bessho, N., TORBert, R. B., Giles, B., Webster, J. et al. 2017 Electron diffusion region during magnetopause reconnection with an intermediate guide field: magnetospheric multiscale observations. J. Geophys. Res. 122, 5235-5246.

Comisso, L. \& Bhattacharjee, A. 2016 On the value of the reconnection rate. J. Plasma Phys. 82, 595820601. 
Dahlburg, R. B., Klimchuk, J. A.\& Antiochos, S. K. 2005 An explanation for the 'switch-on' nature of magnetic energy release and its application to coronal heating. Astrophys. J. 622, $1191-1201$.

Daughton, W. \& Karimabadi, H. 2007 Collisionless magnetic reconnection in large-scale electronpositron plasmas. Phys. Plasmas 14, 072303.

Daughton, W., Roytershteyn, V., Albright, B. J., Karimabadi, H., Yin, L. \& Bowers, K. J. 2009 Transition from collisional to kinetic regimes in large-scale reconnection layers. Phys. Rev. Lett. 103, 065004.

Doss, C. E., Komar, C. M., Cassak, P. A., Wilder, F. D., Eriksson, S. \& Drake, J. F. 2015 Asymmetric magnetic reconnection with a flow shear and applications to the magnetopause. J. Geophys. Res. 120, 7748.

Drake, J. F. \& Lee, Y. C. 1977 Kinetic theory of tearing instabilities. Phys. Fluids 20 (8), 1341.

Drake, J. F., Shay, M. A. \& Swisdak, M. 2008 The hall fields and fast magnetic reconnection. Phys. Plasmas 15, 042396.

DungeY, J. W. 1953 Conditions for the occurrence of electrical discharges in astrophysical systems. Phil. Mag. 44, 725.

Egedal, J., Fox, W., Katz, N., Porkolab, M., Reim, K. \& Zhang, E. 2007 Laboratory observations of spontaneous magnetic reconnection. Phys. Rev. Lett. 98, 015003.

Ergun, R. E., Goodrich, K. A., Wilder, F. D., Holmes, J. C., Stawarz, J. E., Eriksson, S., Sturner, A. P., Malaspina, D. M., Usanova, M. E., Torbert, R. B. et al. 2016 Mms observations of parallel electric fields associated with magnetic reconnection. Phys. Rev. Lett. 116, 235102.

Fletcher, L., Pollack, J. A. \& Potts, H. E. 2004 Tracking of trace ultraviolet flare footpoints. Solar Phys. 222, 279.

Fox, W., Bhattacharjee, A. \& Germaschewski, K. 2011 Fast magnetic reconnection in laserproduced plasma bubbles. Phys. Rev. Lett. 106, 215003.

Furno, I., Intrator, T. P., Hemsing, E. W., Hsu, S. C., Abbate, S., Ricci, P. \& Lapenta, G. 2005 Coalescence of two magnetic flux ropes via collisional magnetic reconnection. Phys. Plasmas 12, 055702.

von Goeler, S., Stodiek, W. \& Sautoff, N. R. 1974 Studies of internal disruptions and $m=1$ oscillations in tokamak discharges with soft-X-ray tecniques. Phys. Rev. Lett. 33, 1201.

Hassam, A. B. 1984 Collisional tearing in field-reversed configurations. Phys. Fluids 27, 2877.

Hesse, M., Schindler, K., Birn, J. \& Kuznetsova, M. 1999 The diffusion region in collisionless magnetic reconnection. Phys. Plasmas 6 (5), 1781.

Hesse, M. \& Zenitani, S. 2007 Dissipation in relativistic pair-plasma reconnection. Phys. Plasmas 14, 112102.

Hesse, M., Zenitani, S., Kuznetsova, M. \& Klimas, A. 2009 A simple, analytical model of collisionless magnetic reconnection in a pair plasma. Phys. Plasmas 16, 102106.

HuAng, Y.-M. \& BhatTACHARJEe, A. 2010 Scaling laws of resistive magnetohydrodynamic reconnection in the high-Lundquist-number, plasmoid unstable regime. Phys. Plasmas 17, 062104.

Huba, J. D., Gladd, N. T. \& Papadopoulos, K. 1977 The lower-hybrid-drift instability as a source of anomalous resistivity for magnetic field reconnection. Geophys. Res. Lett. 4 (3), $125-128$.

Innocenti, M. E., Goldman, M., Newman, D., Markidis, S. \& Lapenta, G. 2015 Evidence of magnetic field switch-off in collisionless magnetic reconnection. Astrophys. J. Lett. 810, L19.

Isobe, H., TAkasaki, H. \& Shibata, K. 2005 Measurement of the energy release rate and the reconnection rate in solar flares. Astrophys. J. 632, 1184.

Isobe, H., Yokoyama, T., Shimojo, M., Morimoto, T., Kozu, H., Eto, S., Narukage, N. \& ShibatA, K. 2002 Reconnection rate in the decay phase of a long duration event flare on 1997 May 12. Astrophys. J. 566, 528.

Jemella, B. D., Shay, M. A., Drake, J. F. \& Rogers, B. N. 2003 Impact of frustrated singularities on magnetic island evolution. Phys. Rev. Lett. 91 (12), 125002. 
Ji, H., Yamada, M., Hsu, S. \& Kulsrud, R. 1998 Experimental test of the sweet-parker model of magnetic reconnection. Phys. Rev. Lett. 80, 3256.

Karimabadi, H., Dorelli, J., Roytershteyn, V., Daughton, W. \& Chacón, L. 2011 Flux pileup in collisionless magnetic reconnection: bursty interaction of large flux ropes. Phys. Rev. Lett. 107, 025002.

Kulsrud, R. M. 2001 Magnetic reconnection: Sweet-Parker versus Petschek. Earth Planets Space 53, 417-422.

Lin, J., Ko, Y.-K., Sui, L., Raymond, J. C., Stenborg, G. A., Jiang, Y., Zhao, S. \& Mancuso, S. 2005 Direct observations of the magnetic reconnection site of an eruption on 2003 November 18. Astrophys. J. 622, 1251.

Liu, Y.-H., Daughton, W., Karimabadi, H., Li, H. \& Gary, S. P. 2014 Do dispersive waves play a role in collisionless magnetic reconnection? Phys. Plasmas 21, 022113.

LiU, Y.-H., DraKe, J. F. \& SWISDAK, M. 2012 The structure of the magnetic reconnection exhaust boundary. Phys. Plasmas 19, 022110.

Liu, Y.-H., Guo, F., Daughton, W., Li, H. \& Hesse, M. 2015 Scaling of magnetic reconnection in relativistic collisionless pair plasmas. Phys. Rev. Lett. 114, 095002.

LIU, Y.-H. \& Hesse, M. 2016 Suppression of collisionless magnetic reconnection in asymmetric current sheets. Phys. Plasmas 23, 060704.

Liu, Y.-H., Hesse, M., Guo, F., Daughton, W., Li, H., Cassak, P. A. \& Shay, M. A. 2017 Why does steady-state magnetic reconnection have a maximum local rate of order 0.1? Phys. Rev. Lett. 118, 085101.

Loureiro, N. F. \& Boldyrev, S. 2017 Role of magnetic reconnection in mhd turbulence. Phys. Rev. Lett. 118, 245101.

Loureiro, N. F., Schekochinin, A. A. \& Cowley, S. C. 2007 Instability of current sheets and formation of plasmoid chains. Phys. Plasmas 14, 100703.

Malakit, K., Shay, M. A., CAssak, P. A. \& BARD, C. 2010 Scaling of asymmetric magnetic reconnection: kinetic particle-in-cell simulations. J. Geophys. Res. 115, A10223.

Mallet, A., Schekochinin, A. A. \& Chandran, B. D. G. 2017 Disruption of sheetlike structures in Alfvénic turbulence by magnetic reconnection. Mon. Not. R. Astron. Soc. 468, 4862.

Mandt, M. E., Denton, R. E. \& Drake, J. F. 1994 Transition to whistler mediated magnetic reconnection. Geophys. Res. Lett. 21, 73.

Matthaeus, W. H. \& LAmKin, S. L. 1985 Rapid magnetic reconnection caused by finite amplitude fluctuations. Phys. Fluids 26, 303.

MCPherron, R. L. 1970 Growth phase of magnetospheric substorms. J. Geophys. Res. 75, 5592.

Melzani, M., Walder, R., Folini, D., Winisdoerffer, C. \& FaVre, J. M. 2014 Relativistic magnetic reconnection in collisionless ion-electron plasmas explored with particle-in-cell simulations. Astron. Astrophys. 570, A111.

MeYer, J. 2013 Structure of the diffusion region in three dimensional magnetic reconnection. PhD thesis, University of Delaware.

Mozer, F. S. \& Pritchett, P. L. 2010 Magnetic field reconnection: a first-principles perspective. Phys. Today 63, 34.

Ng, J., Huang, Y.-M., Hakim, A., Bhattacharjee, A., Stanier, A., Daughton, W., Wang, L. \& GeRMASCHEWSKI, K. 2015 The island coalescence problem: scaling of reconnection in extended fluid models including higher-order moments. Phys. Plasmas 22, 112104.

OHyAmA, M. \& ShibATA, K. 1998 X-ray plasma ejection associated with an impulsive flare on 1992 October 5: physical conditions of x-ray plasma ejection. Astrophys. J. 499, 934.

PARKER, E. N. 1957 Sweet's mechanism for merging magnetic fields in conducting fluids. J. Geophys. Res. 62, 509.

PARKER, E. N. 1963 The solar-flare phenomenon and the theory of reconnection and annihilation of magnetic fields. Astrophys. J. 8, 177.

PARKER, E. N. 1973 The reconnection rate of magnetic fields. Astrophys. J. 180, 247.

PetscheK, H. E. 1964 Magnetic field annihilation. In AAS/NASA Symposium on the Physics of Solar Flares (ed. W. N. Ness), p. 425. NASA. 
Pontin, D. I. 2011 Three-dimensional magnetic reconnection regimes: a review. Adv. Space Res. 47, 1508.

Priest, E. R. \& Forbes, T. R. 2002 The magnetic nature of solar flares. Astron. Astrophs. Rev. 10, 313-377.

QIU, J., LEe, J., GARY, D. E. \& WANG, H. 2002 Motion of flare footpoint emission and inferred electric field in reconnecting current sheets. Astrophys. J. 565, 1335.

Rogers, B. N., Denton, R. E., Drake, J. F. \& Shay, M. A. 2001 Role of dispersive waves in collisionless magnetic reconnection. Phys. Rev. Lett. 87 (19), 195004.

SATO, T. \& HAYASHI, T. 1979 Externally driven magnetic reconnection and a powerful magnetic energy converter. Phys. Fluids 22, 1189.

Servidio, S., Dmitruk, P., Greco, A., Wan, M., Donato, S., Cassak, P. A., Shay, M. A., Carbone, V. \& Matthaeus, W. H. 2011 Magnetic reconnection as an element of turbulence. Nonlinear Processes Geophys. 18, 675.

Servidio, S., Matthaeus, W. H., Shay, M. A., Cassak, P. A. \& Dmitruk, P. 2009 Magnetic reconnection in two-dimensional magnetohydrodynamic turbulence. Phys. Rev. Lett. 102, 115003.

ShaY, M. A. \& Drake, J. F. 1998 The role of electron dissipation on the rate of collisionless magnetic reconnection. Geophys. Res. Lett. 25, 3759.

Shay, M. A., Drake, J. F., Rogers, B. N. \& Denton, R. E. 1999 The scaling of collisionless, magnetic reconnection for large systems. Geophys. Res. Lett. 26 (14), 2163-2166.

Shay, M. A., Drake, J. F. \& SwISDAK, M. 2007 Two-scale structure of the electron dissipation region during collisionless magnetic reconnection. Phys. Rev. Lett. 99, 155002.

Shay, M. A., Drake, J. F., Swisdak, M. \& Rogers, B. N. 2004 The scaling of embedded collisionless reconnection. Phys. Plasmas 11, 2199.

SHEPHERD, L. S. \& CASSAK, P. A. 2010 Comparison of secondary islands in collisional reconnection to Hall reconnection. Phys. Rev. Lett. 105, 015004.

Shibata, K. \& TANuma, S. 2001 Plasmoid-induced-reconnection and fractal reconnection. Earth Planets Space 53, 473.

Simakov, A. N., Chacón, L. \& KnOll, D. 2006 Semi-analytical model for flux-pileup-limited, dynamically reconnecting systems in resistive magnetohydrodynamics. Phys. Plasmas 13, 082103.

Sironi, L., Giannios, D. \& Petropoulou, M. 2016 Plasmoids in relativistic reconnection, from birth to adulthood: first they grow, then they go. Mon. Not. R. Astron. Soc. 462, 48.

Smith, D., Ghosh, S., Dmitruk, P. \& Matthaeus, W. H. 2004 Hall and turbulence effects on magnetic reconnection. Geophys. Res. Lett. 31, L02805.

SonNeruP, B. U. Ö. 1979 Magnetic field reconnection. In Solar System Plasma Physics (ed. L. J. Lanzerotti, C. F. Kennel \& E. N. Parker), vol. 3, p. 46. North Holland Pub.

Stanier, A., Daughton, W., Chacón, L., Karimabadi, H., NG, J., Huang, Y.-M., Hakim, A. \& BHATTACHARJEe, A. $2015 a$ Role of ion kinetic physics in the interaction of magnetic flux ropes. Phys. Rev. Lett. 115, 175004.

Stanier, A., Simakov, A. N., Chacón, L. \& Daughton, W. $2015 b$ Fast magnetic reconnection with large guide fields. Phys. Plasmas 22, 010701.

Strauss, H. R. 1987 Turbulent reconnection. Astrophys. J. 326, 412.

Swisdak, M., Drake, J. F., Shay, M. A. \& Rogers, B. N. 2003 Diamagnetic suppression of component magnetic reconnection at the magnetopause. J. Geophys. Res. 108, 1218.

SWISDAK, M., LIU, Y. H. \& DRAKE, J. F. 2008 Development of a turbulent outflow during electronpositron magnetic reconnection. Astrophys. J. 680, 999.

Terasawa, T. 1983 Hall current effect on tearing mode instability. Geophys. Res. Lett. 10, 475.

Trintchouk, F., Yamada, M., Ji, H., Kulsrud, R. M. \& CARTer, T. A. 2003 Measurement of the transverse spitzer resistivity during collisional magnetic reconnection. Phys. Plasmas 10 (1), 319-322.

UGAI, M. 1984 Self-consistent development of fast magnetic reconnection with anomalous resistivity. Plasma Phys. Control. Fusion 26 (12B), 1549-1563. 
Ugai, M. \& Tsuda, T. 1977 Magnetic field line reconnexion by localized enhancement of resistivity, 1, evolution in a compressible mhd fluid. J. Plasma Phys. 17, 337.

UZDENSKY, D. A. 2007 The fast collisionless reconnection condition and the self-organization of solar coronal heating. Astrophys. J. 671, 2139.

Uzdensky, D. A. \& Kulsrud, R. M. 2000 Two-dimensional numerical simulation of the resistive reconnection layer. Phys. Plasmas 7 (10), 4018.

Wang, S., Kistler, L. M., Mouikis, C. G. \& Petrinec, S. M. 2015 Dependence of the dayside magnetopause reconnection rate on local conditions. J. Geophys. Res. 120, 6386.

Yamada, M., Kulsrud, R. \& Ji, H. 2010 Magnetic reconnection. Rev. Mod. Phys. 82, 603.

Yokoyama, T., Akita, K., Morimoto, T., Inoue, K. \& Newmark, J. 2001 Clear evidence of reconnection inflow of a solar flare. Astrophys. J. 546, L69.

Zenitani, S. \& Hesse, M. 2008 The role of the weibel instability at the reconnection jet front in relativistic pair plasma reconnection. Phys. Plasmas 15, 022101.

Zweibel, E. G. \& YAmadA, M. 2009 Magnetic reconnection in astrophysical and laboratory plasmas. Annu. Rev. Astron. Astrophys. 47, 291. 